\title{
Fatty acid oxidation is associated with proliferation and prognosis in breast and other cancers
}

\author{
Aziz Aiderus, Michael A. Black and Anita K. Dunbier *i]
}

\begin{abstract}
Background: Altered cellular metabolism is a hallmark of cancer but the association between utilisation of particular metabolic pathways in tumours and patient outcome is poorly understood. We sought to investigate the association between fatty acid metabolism and outcome in breast and other cancers.

Methods: Cox regression analysis and Gene Set Enrichment Analysis (GSEA) of a gene expression dataset from primary breast tumours with well annotated clinical and survival information was used to identify genesets associated with outcome. A geneset representing fatty acid oxidation (FAO) was then examined in other datasets. A doxycyclineinducible breast cancer cell line model overexpressing the rate-limiting enzyme in $\mathrm{FAO}$, carnitine palmitoyl transferase 1A (CPT1A) was generated and analysed to confirm the association between FAO and cancer-associated characteristics in vitro.

Results: We identified a gene expression signature composed of 19 genes associated with fatty acid oxidation (FAO) that was significantly associated with patient outcome. We validated this observation in eight independent breast cancer datasets, and also observed the FAO signature to be prognostic in other cancer types. Furthermore, the FAO signature expression was significantly downregulated in tumours, compared to normal tissues from a variety of anatomic origins. In breast cancer, the expression of CPT1A was higher in oestrogen receptor (ER)-positive, compared to ER-negative tumours and cell lines. Importantly, overexpression of CPT1A significantly decreased the proliferation and wound healing migration rates of MDA-MB231 breast cancer cells, compared to basal expression control.
\end{abstract}

Conclusions: Our findings suggest that FAO is downregulated in multiple tumour types, and activation of this pathway may lower cancer cell proliferation, and is associated with improved outcomes in some cancers.

Keywords: Fatty acid oxidation, Gene signature, Cancer, Prognosis, Tumour-normal, CPT1A, Proliferation, Migration

\section{Background}

Improved understanding of the molecular features associated with prognosis in primary tumours is key to better management of the disease. High throughput gene expression technologies have facilitated the molecular profiling of tumours and generation of prognostic gene signatures [1]. However, particularly in breast cancer, many of these gene signatures contain genes that are strongly correlated with proliferation, and the biology underlying their enrichment is poorly understood $[2,3]$.

\footnotetext{
* Correspondence: anita.dunbier@otago.ac.nz

Centre for Translational Cancer Research and Department of Biochemistry, University of Otago, Dunedin 9054, New Zealand
}

Alterations in cellular metabolism and energetics are hallmarks of cancer $[4,5]$. One of the earliest observations of altered tumour metabolism was increased aerobic glycolytic flux, termed the Warburg effect [6]. A large number of studies focusing on this pathway have subsequently found that glycolysis serves energetic and anabolic roles for cell division [7]. Technological advances such as metabolomics and isotope tracing have been employed to study cellular metabolism and have revealed that other metabolic pathways that are co-opted by tumours to support cancer cell division [8]. For example, some tumours have increased reliance on the oxidation of the amino acid glutamine [8], however, the role of fatty acid metabolism in cancer remains

(C) The Author(s). 2018 Open Access This article is distributed under the terms of the Creative Commons Attribution 4.0 International License (http://creativecommons.org/licenses/by/4.0/), which permits unrestricted use, distribution, and 
unclear. Specifically, while the role of fatty acid synthesis is better understood, how FAO affects tumour biology remain contentious [9]. Furthermore, even though most of these metabolic pathways have been extensively studied using in vitro and in vivo systems, their association with patient outcome remains to be determined.

In this study, we report the generation and validation of a gene signature involved in fatty acid oxidation (FAO) and prognosis in breast, and several other cancer types. Our findings suggest that pharmacologic agents that upregulate FAO may have therapeutic potential.

\section{Methods}

Cox regression analysis and gene set enrichment analysis Gene expression data and associated clinical information from the METABRIC study [10] was obtained through Sage Bionetworks with appropriate ethical approval (University of Otago Human Ethics Approval H16/092) and was used as the training dataset. All data analysis was performed using the R Software [11]. Only patients with ER-positive tumours that received radiation and/or endocrine therapy $(n=973)$ were included. Gene expression data was collapsed so that each gene was represented by a single probe using the collapseRows function from the 'WGCNA' package [12]. Cox regression analysis was performed using the coxph function available from the 'survival' package [13]. The $p$ values associated with the hazard ratios for each gene were adjusted for multiple comparisons by the false discovery rate (FDR) method [14]. Genes and associated $p$ values were then sorted in ascending order (most-to-least significant) and pre-ranked gene set enrichment analysis [15] was performed using the KEGG database [16]. Hierarchical clustering and heatmaps were generated using the heatmap. 2 function with Euclidean as the distance metric and complete linkage as the linkage criterion.

Survival, multivariable Cox regression, and logistic regression analyses - All survival analyses were performed in RStudio using the 'survival' package, or using the KMplotter online software [13, 17]. Statistical significance for differences between survival curves was calculated using the log-rank test [13]. Multivariable Cox regression analysis was conducted using available clinico-pathologic factors, depending on the datasets analysed. For survival analysis, the average expression of the 19-gene fatty acid oxidation signature was calculated for each patient, and stratified into two groups - above or below the median. For validation analysis on independent breast cancer datasets, the log-rank $p$ values were adjusted for multiple comparisons using the FDR method. To estimate the odds-ratio of achieving pathologic complete response to neoadjuvant chemotherapy based on low (below median) or high (above median) expression of the fatty acid oxidation signature expression, logistic regression was performed. The final meta-analysis odds ratio was obtained by taking the average value of the point estimates and confidence intervals. The datasets used for the validation analysis of the fatty acid oxidation signature, conducting logistic regression on neoadjuvant chemotherapy breast cancer trials, and tumour-normal analysis are summarised in Additional file 1: Table S1.

In silico CPT1A expression analysis in breast tumours and cell lines - Datasets used for validation analysis of the FAO signature were also used to investigate the expression of CPT1A in breast tumours. For breast cancer cell lines, two datasets were analysed for expression of CPT1A: (i) quantile normalised with gene level summary data [18] from E-MTAB-181 [19] was accessed from ArrayExpress, and (ii) GSE57083 accessed from NCBI GEO where the RMA-normalised [20] expression matrix was used to calculate the average expression of CPT1A for each cell line based on the values from four probesets: 203633_at, 203634_s_at, 210687_at and 210688_s_at on the Affymetrix Human Genome U133 Plus 2.0 array.

CPT1A overexpression in MDA-MB231 cell line - The coding sequence for CPT1A (NM_001876.3) was accessed from the NCBI Nucleotide portal and primers were designed to amplify the entire sequence. Total RNA from MCF10A normal mammary epithelial cells were converted to CDNA, and high-fidelity PCR performed to amplify the CPT1A coding sequence. PCR products were gel-purified, digested with $S a c \mathrm{II}$ and $\mathrm{XbaI}$, and ligated into a doxycycline-inducible plasmid downstream of a Tet-response element (TRE). The CPT1A coding sequence was Sanger sequenced to verify that no mutations were introduced during the cloning procedure.

Doxycycline dose response and time course characterisation - For dose response and time course analysis of doxycycline (Dox)-induced CPT1A expression, selected MDA-MB231 pTRE-CPT1A clones were seeded in 6 well plates and induced with $2 \mu \mathrm{g} / \mathrm{mL}$ Dox for $48 \mathrm{~h}$. Whole cell lysates were prepared and resolved by SDS-PAGE. Proteins were transferred to a PVDF membrane, blocked for one hour with $5 \%(w / v)$ milk, and incubated with a CPT1A antibody (Abcam 128,568, mouse anti-human, $1 \mu \mathrm{g} / \mathrm{mL}$ ) overnight. Membranes were washed three times with $1 \mathrm{X}$ TBST solution for 10 mins per wash, and then incubated with secondary antibody (Amersham NA931V, sheep anti-mouse 1:10,000) for one hour at room temperature. Membranes were washed as described above, and incubated with $2 \mathrm{~mL}$ of enhanced chemiluminescent solution for 5 mins, prior to imaging on the Odyssey LiCor system.

Proliferation rate analysis between basal and CPT1A overexpression in MDA-MB231 cell line - To investigate whether CPT1A overexpression affects the proliferation rate of MDA-MB231 cells, TetOn parental and pTRE-CPT1A clones were seeded in 6 well plates and induced with $2 \mu \mathrm{g} /$ 
$\mathrm{mL}$ Dox for $48 \mathrm{~h}$. Cells were then seeded in 96 well plates (1000 cells/well) with and without Dox, and real-time proliferation was monitored using the IncuCyte live cell imaging system (Essen Bioscience).

Wound healing rate analysis between basal and CPT1A overexpression in MDA-MB231 cell line - To compare the wound healing migration rates between basal and CPT1A overexpression in MDA-MB231 cells, TetOn parental and pTRE-CPT1A clones were seeded in 6 well plates and induced with Dox for 5 days. Cells were then seeded in an Essen ImageLock 96-well plate at full confluency $(60,000$ cells/well). The following day, a scratch was created through the middle of each well using the Essen WoundMaker and fresh media was replaced in each well. Real time wound healing migration rates were monitored using the IncuCyte live cell imaging system.

Soft agar clonogenic growth analysis between basal and CPT1A overexpression in MDA-MB231 cell line - To investigate whether CPT1A overexpression affects anchorage-independent colony formation in MDA-MB231 cells, TetOn parental and pTRE-CPT1A clones were seeded in $0.3 \%$ agar and layered over $0.6 \%$ agar. The soft agar assay was conducted for two weeks, with media changes every 2-3 days. Cells were then fixed with $10 \%$ methanol, stained with $0.1 \%$ crystal violet and the number of colonies counted under a phase contrast microscope.

\section{Results}

Cox regression and gene set enrichment analysis to identify genes associated with breast cancer disease-specific survival - To identify genes and pathways that are significantly associated with disease-specific survival in the METABRIC cohort, we performed Cox regression analysis on gene expression data from 973 primary breast tumours from the METABRIC study. The resulting $p$-values were adjusted using the false discovery rate (FDR) method. The genes were then sorted according to their adjusted $p$-values, and pre-ranked GSEA analysis using the KEGG database was performed.

Table 1 summarises the KEGG pathways that were significantly enriched in the Cox regression analysis. Several of the enriched pathways such as 'DNA replication,' 'Pyrimidine Metabolism' and 'Base Excision Repair' contain genes correlated with proliferation, which has been shown to be highly prognostic in ER-positive breast cancer $[21,22]$.

For further investigation we focused on the KEGG Fatty Acid Metabolism pathway, which was one of the gene sets that was enriched (nominal $p=0.002$, FDR adjusted $q=0.03$ ) (Fig. 1a). Of note, 19 out of 42 genes in this gene set were defined as core-enriched based on the output of the analysis, which means they were over-represented at the top of the pre-ranked gene list provided and contributed the most to the enrichment of this pathway. These 19 genes are referred to as the "fatty acid oxidation (FAO)" signature hereafter (Table 2). Included in this signature were genes which have previously been identified as the core components of the fatty acid beta-oxidation pathway, such as CPT1A, CPT2, $A C A D M, A C A D S B$, and $A C A D V L$ [23].

Hierarchical clustering analysis revealed that patients with relatively high or low expression of the FAO signature (denoted as 'FAO average score' horizontal bar above the heatmap) were clustered together toward the left or the right of the heatmap, respectively (Fig. 1a). Based on their average FAO signature value, patients in this cohort were stratified into two groups (one made up of patients with expression higher than the median value, and one of patients with expression lower than the median value), and Kaplan-Meier survival curve analysis revealed patients with tumours with high expression (above median cutoff) of the FAO signature had a significantly better outcome (Fig. 1b, log-rank $p=4.40 \mathrm{e}-06$ ). This finding was validated in eight independent datasets, and patients in the low group had univariate hazard ratios between 1.3 and 5.4 for indicated survival metrics (Table 3). These associations

Table 1 KEGG pathways associated with disease-specific survival in METABRIC training cohort

\begin{tabular}{lllll}
\hline KEGG pathways & Enrichment score & Normalised enrichment score & Nominal $p$-value & FDR q value \\
\hline Valine, leucine, isoleucine degradation & 0.53 & 1.74 & 0 & 0.03 \\
Base excision repair & 0.55 & 1.71 & 0 & 0.03 \\
Pyrimidine metabolism & 0.48 & 1.68 & 0 & 0.03 \\
DNA replication & 0.52 & 1.66 & 0.002 & 0.03 \\
Glyoxylate and dicarboxylate metabolism & 0.58 & 1.63 & 0 & 0.03 \\
Oocyte meiosis & 0.46 & 1.62 & 0.002 & 0.03 \\
Glycine, serine, threonine metabolism & 0.51 & 1.61 & 0.002 & 0.03 \\
Proteasome & 0.48 & 1.58 & 0.002 & 0.04 \\
Fatty acid metabolism & 0.48 & 1.58 & 0.04
\end{tabular}

Proliferation associated pathways are in bold, while the Fatty Acid Metabolism pathway analysed further in this study is in italic 

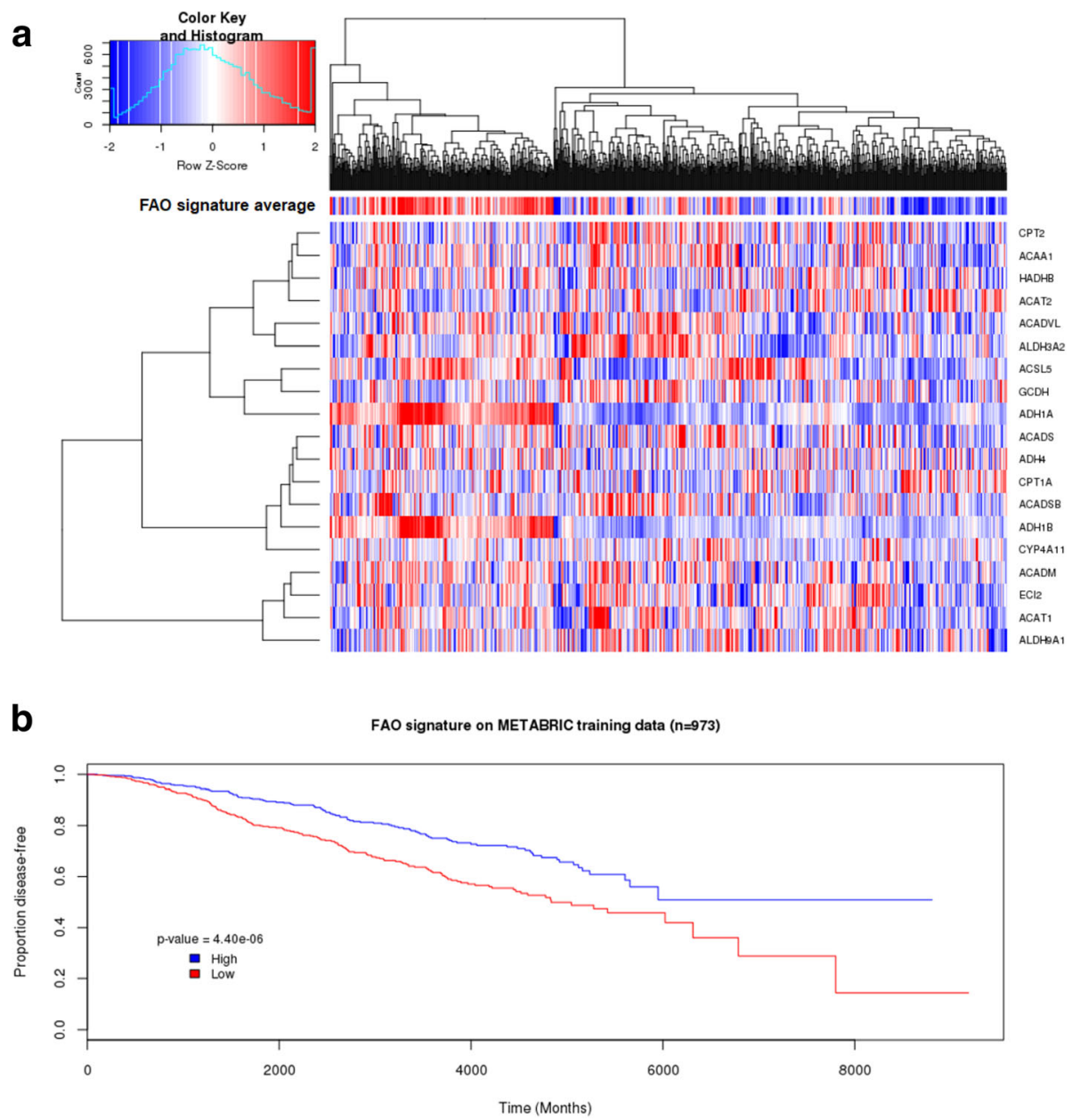

Fig. 1 Expression of the 19-gene FAO signature is prognostic in the METABRIC training cohort. a Heatmap depicting expression of the FAO signature in the METABRIC training cohort. Rows correspond to the expression of indicated genes, while columns correspond to each patient ( $n=973$ ). Red and blue correspond to low and high gene expression respectively, on a continuous scale.b Kaplan-Meier survival curve of FAO signature expression in the METABRIC training cohort. Outcomes in patients with high (blue) expression of the FAO signature differs significantly than patients in the low (red) group (log-rank $p=4.40 \mathrm{e}-06$ ). High and low expression was defined as patients with average FAO signature expression value above and below the median cutoff, respectively

were statistically significant in cohorts with distant relapse-free or metastasis-free survival data available. Additionally, the FAO signature expression was also significantly associated with overall survival in two cohorts, and trended towards significance in the TCGA breast cohort (adjusted $p=0.097$, univariate hazard ratio 1.31 (95\% confidence interval 0.95-1.81)). Taken together, these data suggest a robust association between expression of genes involved in FAO and prognosis in breast cancer.

FAO signature expression is negatively correlated with proliferation - As low expression of the FAO signature expression is associated with poor outcome in breast cancer patients, and patients with highly proliferative tumours typically have a poor prognosis, we investigated whether the FAO signature expression was correlated with proliferation. Spearman correlation analysis between the
FAO and the mitosis kinome score (MKS) [22] - an 11-gene proliferation signature - found a significant, negative correlation between the two signatures in all six cohorts (Spearman's rho $=-0.27$ to -0.6 ), suggesting that the FAO signature expression is inversely correlated with tumour proliferation (Table 4).

Low FAO signature expression is correlated with clinical features associated with poor prognosis - We then explored the association between the FAO signature expression and various clinical features. The FAO signature was significantly higher in ER-positive, compared to negative tumours (Figs 2a, b, Wilcoxon rank sum test $p<0.01$ ); grade 1 compared to 3 (Figs 2c, d, Wilcoxon rank sum test $p<0.01$ ); and luminal compared to basal/ HER2-enriched molecular subtypes (Figs 2e, f, g, Wilcoxon rank sum test $p<0.01)$. Hence, the findings suggest 
Table 2 Gene symbols and names for members of the 19-gene FAO signature

\begin{tabular}{|c|c|}
\hline $\begin{array}{l}\text { Gene } \\
\text { symbol }\end{array}$ & Gene name \\
\hline$\overline{A C A A 1}$ & acetyl CoA acyltransferase 1 \\
\hline CPT1A & carnitine palmitoyl transferase $1 \mathrm{~A}$ \\
\hline ACADM & acyl CoA dehydrogenase, C-4 to C-12 straight chain \\
\hline GCDH & glutaryl-CoA dehydrogenase \\
\hline ACADS & acyl CoA dehydrogenase, $C-2$ to $C-3$ straight chain \\
\hline ACAT2 & acetyl CoA acetyltransferase 2 \\
\hline $\mathrm{ECl} 2$ & enoyl CoA isomerase 2 \\
\hline ACAT1 & acetyl CoA acetyltransferase 1 \\
\hline ACADSB & acyl CoA dehydrogenase, short/branched chain \\
\hline CYP4A11 & cytochrome P450 family 4 subfamily A member 11 \\
\hline ACADVL & acyl CoA dehydrogenase, very long \\
\hline $\mathrm{ADH} 1 \mathrm{~A}$ & alcohol dehydrogenase 1A, (class I), alpha polypeptide \\
\hline CPT2 & carnitine palmitoyl transferase 2 \\
\hline $\mathrm{HADHB}$ & $\begin{array}{l}\text { hydroxyacyl CoA dehydrogenase/3-ketoacyl CoA thiolase/ } \\
\text { enoyl CoA hydratase, trifunctional protein beta subunit }\end{array}$ \\
\hline $\mathrm{ADH} 1 \mathrm{~B}$ & alcohol dehydrogenase $1 \mathrm{~B}$, (class I), beta polypeptide \\
\hline ALDH9A1 & alcohol dehydrogenase 9, family member A1 \\
\hline ACSL5 & acyl-CoA synthetase long-chain family member 5 \\
\hline $\mathrm{ADH} 4$ & alcohol dehydrogenase 4 (class II), pi polypeptide \\
\hline ADLH3A2 & aldehyde dehydrogenase 3 family member A2 \\
\hline
\end{tabular}

that the FAO signature is associated with clinical features that are linked to poor prognosis.

FAO signature expression is prognostic, independent of standard histopathologic features in breast cancer Next, we investigated whether the FAO signature expression provided prognostic information that was independent of standard histopathologic variables. To achieve this, we performed multivariable Cox regression analysis on seven independent breast tumour gene expression datasets.

As summarised in Table 5, after including available established prognostic factors such as tumour grade, size, lymph node and ER status in the Cox model, the FAO signature expression provides significant, independent prognostic information, with patients in the Low group having hazard ratios that range from 1.5 to 5.5 , relative to patients in the High group.

FAO signature expression is associated with favourable response to short-term, neoadjuvant chemotherapy or aromatase inhibition - In the previous analyses, several datasets that were used to validate the prognostic performance of the FAO signature included patients who received adjuvant treatment. To determine whether the FAO signature expression is associated with neoadjuvant endocrine or chemotherapy response, logistic regression was performed.

As shown in Fig. 3a, pre-treatment tumour samples from 102 patients [24] who received short term oestrogen deprivation treatment and achieved complete response as determined by the Response Evaluation Criteria In Solid Tumours (RECIST) had higher expression of the FAO signature, compared to patients who progressed (Wilcox rank sum test $p=0.002$ ). We also determined the odds ratio between the FAO signature expression and pathologic complete response ( $\mathrm{pCR}$ ) to chemotherapy using published gene expression data from six neoadjuvant chemotherapy trials [25-30]. As summarised in Fig. 3b, patients with low FAO signature expression had greater odds of achieving pCR, compared to patients in the high group (average odds ratio 2.94, 95\% confidence interval 1.38-6.82). Taken together, these data suggest that the FAO signature expression in primary breast tumours is associated with response to both neoadjuvant chemotherapy and aromatase inhibitor therapy.

Table 3 FAO signature expression is prognostic in independent breast cancer datasets

\begin{tabular}{|c|c|c|c|c|c|c|}
\hline Dataset & Study & $\mathrm{n}$ & $\begin{array}{l}\text { Log rank } p \\
\text { (survival metric) }\end{array}$ & $\begin{array}{l}\text { Hazard ratio } \\
\text { (Low vs. High) }\end{array}$ & $\begin{array}{l}\text { Hazard ratio } \\
\text { ( } 95 \% \text { confidence interval) }\end{array}$ & FDR adjusted $p$ \\
\hline \multirow[t]{2}{*}{ GSE42568 } & Clarke et al. & 104 & 0.00562 (RFS) & 2.24 & $1.25-4.03$ & 0.007 \\
\hline & & 104 & 0.0000958 (OS) & 4.05 & $1.84-8.66$ & 0.000311 \\
\hline \multirow[t]{2}{*}{ GSE20685 } & Kao et al. & 327 & 0.0118 (DMFS) & 1.98 & $1.26-3.11$ & 0.003 \\
\hline & & 327 & 0.00243 (OS) & 1.75 & $1.12-2.27$ & 0.013 \\
\hline GSE46563 & Jonsdottir et al. & 94 & 0.000543 (DMFS) & 5.46 & $1.86-16.1$ & 0.002 \\
\hline GSE25066 & Hatzis et al. & 508 & $5.98 \mathrm{E}-06$ & 2.43 & $1.64-3.62$ & $1.17 \mathrm{E}-05$ \\
\hline \multirow[t]{2}{*}{ TCGA BRCA } & TCGA breast cancer & 776 & 0.00387 (RFS) & 2.08 & $1.25-3.47$ & 0.0047 \\
\hline & & 1096 & 0.097 (OS) & 1.31 & $0.95-1.81$ & 0.097 \\
\hline GSE22219 & Buffa et al. & 216 & 0.00753 (DRFS) & 1.82 & $1.17-2.85$ & 0.0085 \\
\hline BRCA2116 & Nagalla et al. & 672 & 6.51E-05 (DRFS) & 2.02 & $1.42-2.88$ & $9.10 \mathrm{E}-05$ \\
\hline GSE21653 & Sabatier et al. & 266 & 0.03 (DFS) & 1.64 & $1.06-2.55$ & 0.03 \\
\hline
\end{tabular}


Table 4 FAO signature expression is negatively correlated with proliferation

\begin{tabular}{lllll}
\hline Dataset & Study & $\mathrm{n}$ & Spearman's rho & $p$ \\
\hline GSE42568 & Clarke et al. & 104 & -0.27 & $5.17 \mathrm{E}-03$ \\
GSE20685 & Kao et al. & 327 & -0.45 & $<3.85 \mathrm{E}-16$ \\
GSE46563 & Jonsdottir et al. & 94 & -0.4 & $7.70 \mathrm{E}-15$ \\
GSE25066 & Hatzis et al. & 508 & -0.45 & $<3.85 \mathrm{E}-16$ \\
GSE22219 & Buffa et al. & 216 & -0.6 & $<3.85 \mathrm{E}-16$ \\
TCGA BRCA & TCGA breast cancer & 1215 & -0.59 & $<3.85 \mathrm{E}-16$ \\
\hline
\end{tabular}

Spearman's correlation analysis between the FAO and MKS proliferation gene signature in breast cancer datasets

Expression of FAO signature is prognostic in other cancer types - To investigate whether the prognostic performance of the FAO signature could be extended to other tumour types, we explored whether the signature expression was associated with prognosis in an additional six different cancer types.

The FAO signature was highly prognostic in gastric (Fig. 4a, log-rank $p=8.9 \mathrm{e}-09$ ) and lung adenocarcinoma (Fig. 4b, log-rank $p<1.1 \mathrm{e}-16$ ). We also analysed clear cell renal cell carcinoma (ccRCC) and melanoma cohorts from TCGA and observed high FAO signature expression to be associated with better prognosis (Fig. 4c, ccRCC log-rank $p=3.7$ e-07; Fig. 4d, melanoma $\log$-rank $p=0.042$ ). However, in colorectal and ovarian cancers, no significant differences in survival were observed between the FAO signature expression and outcome (Additional file 2: Table S2, Additional file 3: Fig. S1). These data suggest that the FAO signature expression is prognostic in several other different cancer types, in addition to breast cancer.

FAO signature expression is lower in tumour, compared to non-cancerous tissues - The observed association between the FAO signature and prognosis in some cancer types raises the question as to whether expression of this signature is altered in tumour, compared to non-tumour or normal tissues. To address this question, we analysed the FAO signature expression across tumour and non-tumour tissues using publicly available gene expression datasets from multiple cancer types. As shown in Fig. 5, the FAO signature expression was consistently downregulated in tumours, compared to non-tumour tissues in all datasets analysed. In prostate cancer, we observed a trend of decreased expression of the FAO signature between non-tumour and primary tumour tissues, which achieved statistical significance when the former was compared to metastatic tissues (Fig. 5b). In oral cancer, the FAO signature expression was lower in benign dysplasia and primary tumours, compared to non-tumour tissues (Fig. 5h). These data suggest that tumours from various anatomic sites downregulate the expression of the FAO signature compared to normal, healthy tissues.

Expression of CPT1A is expressed higher in ER-positive, compared to ER-negative breast tumours and cell lines Based on our computational findings, we sought to understand how modulating FAO affects breast cancer cell biology. Since CPT1A is a member of the 19-gene signature and the rate-limiting enzyme in FAO, we modulated the expression of this enzyme in vitro. Of note, genetic modulation and pharmacologic inhibition of CPT1A has been shown to alter FAO flux [31-33]. Since low FAO signature expression in breast tumours was associated with poor outcome, we investigated the effect of CPT1A overexpression in a breast cancer cell line.

First, we surveyed the expression levels of CPT1A in breast cancer tissues and cell lines. Five out of six primary breast tumour gene expression datasets (details in Additional file 1: Table S1) analysed showed significantly lower CPT1A expression in ER-negative, compared to ER-positive tumours (Figs 6a-f). Consistent with the findings observed in tumour tissues, analysis of
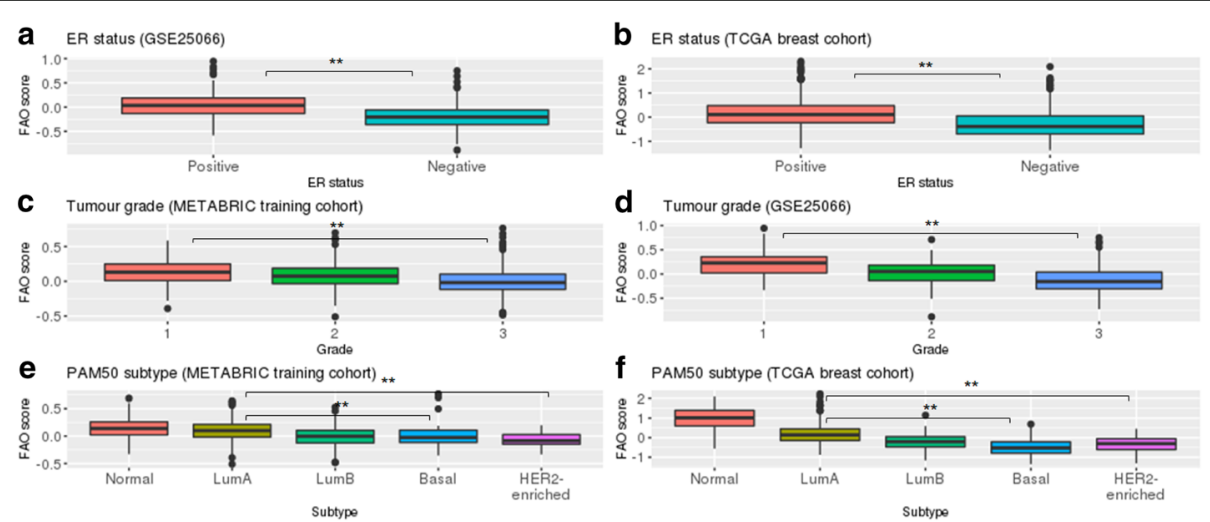

Fig. $\mathbf{2}$ Low FAO signature expression is correlated with clinical features associated with poor prognosis. FAO signature expression is lower in $\mathbf{a}$, $\mathbf{b}$ ER-negative, $\mathbf{c}$, $\mathbf{d}$ higher tumour grade, and $\mathbf{e}, \mathbf{g}$ basal-like and HER2-enriched molecular subtypes in different breast cancer datasets. ** Wilcoxon rank sum test $p<0.01$ 
Table 5 FAO signature expression is prognostic independent of standard histopathological features in breast cancer

\begin{tabular}{llllll}
\hline Dataset & Study & $\mathrm{n}^{\mathrm{a}}$ & Covariates & Hazard ratio (survival metric) & FDR adjusted $p$ \\
\hline GSE25066 & Hatzis et al. & 466 & Grade, ER status & 1.62 (DRFS) & 0.0592 \\
BRCA2116 & Nagalla et al. & 660 & Grade, size, lymph node status & 1.5 (DRFS) & 0.09 \\
GSE42568 & Clarke et al. & 104 & Grade, size, lymph node status & 2.55 (RFS) & 0.009 \\
GSE22219 & Buffa et al. & 216 & ER status, size, age, lymph node status & 1.91 (DRFS) & 0.014 \\
GSE46563 & Jonsdottir et al. & 94 & Grade, ER status, size & 5.54 (DRFS) & 0.009 \\
GSE20685 & Kao et al. & 327 & Age & 1.7 (DRFS) & 0.009 \\
TCGA BRCA & TCGA breast cancer & 776 & ER status & 1.61 (RFS) & 0.09 \\
\hline
\end{tabular}

af note, the sample sizes in this analysis differ slightly with that presented in Table 3 as there were incomplete clinical information for some patients in certain datasets. DRFS, distant relapse-free survival; $R F S$, relapse-free survival

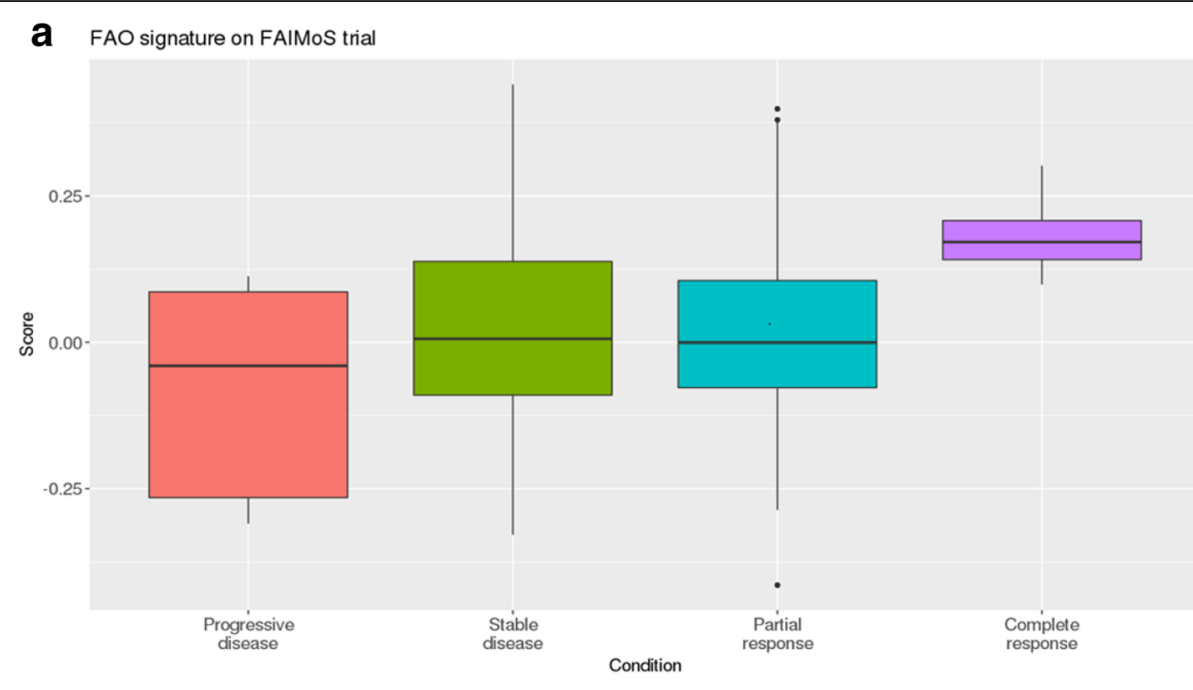

b

\section{Study}

Shi et al. (2010)

Hatzis et al. (2011)

Iwamoto et al. (2011)

Miyake et al. (2012)

Shen et al. (2012)

Hess et al. (2006)

Total
FAO signature and breast cancer chemotherapy response
PCR NOPCR

$56 / 278$
$99 / 508$
$20 / 61$
$26 / 115$
$37 / 91$
$34 / 133$

$272 / 1186$

Odds Ratio $(95 \% \mathrm{Cl}) \quad$ Odds Ratio $(95 \% \mathrm{Cl})$

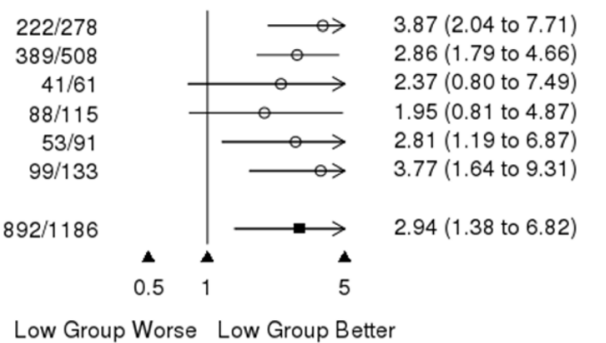

Fig. 3 FAO signature expression is associated with response to short-term, neoadjuvant oestrogen deprivation or chemotherapy in breast tumours. a High FAO signature expression in pre-treatment primary breast tumours is associated with complete response, compared to progressive disease (Complete response vs Progressive disease Wilcoxon rank sum test $p=0.002$ ). b Odds ratio of achieving pathological complete response ( $p C R$ ) based on the FAO signature expression for each neoadjuvant chemotherapy trial indicated 

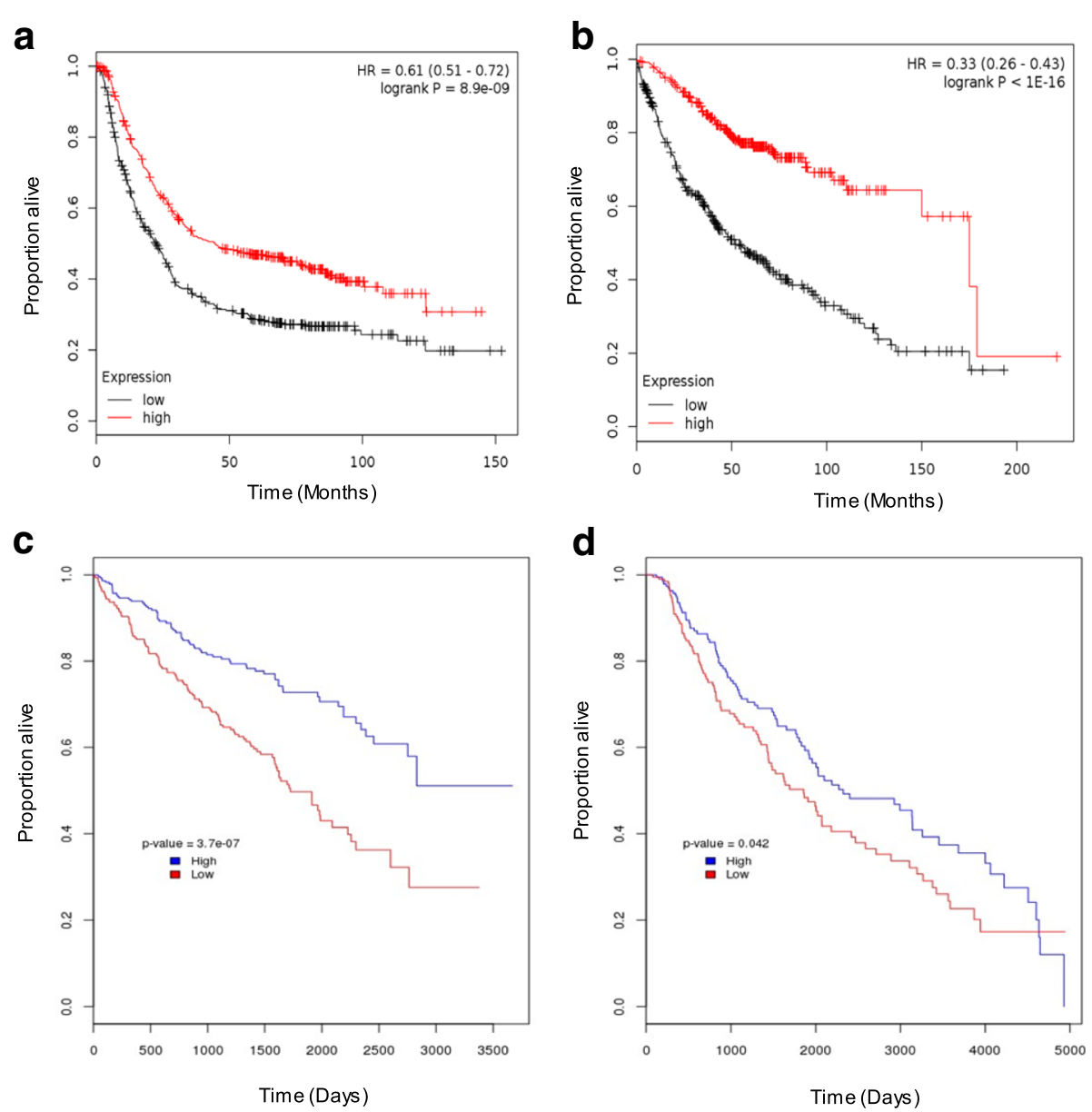

Fig. $4 \mathrm{FAO}$ signature expression is prognostic in different cancers. High expression of the FAO signature in a gastric $(n=876$, log-rank $p=8.9 \mathrm{E}-$ $09)$ and $\mathbf{b}$ lung adenocarcinoma $(n=720$, log-rank $p<1.1 \mathrm{E}-16)$ is associated with good overall survival in the KMplotter cohort. High expression of the FAO signature in the TCGA c cCRCC $(n=588$, log-rank $p=3.7 \mathrm{E}-07)$ and $\mathbf{d}$ melanoma $(n=396, \log$-rank $p=0.042)$ cohorts is correlated with good overall survival. High and low expression was defined as patients with average FAO signature expression value above and below the median cutoff, respectively

published microarray gene expression data from a panel of breast cancer cell lines revealed a striking enrichment of lower CPT1A expression in ER-negative, compared to ER-positive cell lines (Fig. 6g Wilcoxon rank sum test $p$ $=5.41 \mathrm{e}-10$; Fig. $6 \mathrm{~h}$ Wilcoxon rank sum test $p=3.49 \mathrm{e}-09$ ). This finding was also observed in an independent dataset, and additionally, CPT1A expression was decreased in an MCF7 cell line that is resistant to the chemotherapeutic agent adriamycin (Additional file 4: Fig. S2, black arrow), compared to the wildtype parental line. Based on data from the in silico analysis, the ER-negative MDA-MB231 cell line, which has low mRNA expression of CPT1A, was selected for overexpression analysis.

Overexpression of CPT1A decreases confluency in MDA-MB231 cell line - As we had observed that the expression of the FAO signature is inversely correlated with proliferation in tumours, we investigated whether increased CPT1A expression altered the rate at which cultured cells achieved confluency. We generated a doxycycline-inducible system to overexpress CPT1A in the MDA-MB231 cell line [34, 35]. Characterisation of the induction of CPT1A by western blot analysis is this shown in Additional file 5: Fig. S3. Cells were pre-induced with doxycycline for $48 \mathrm{~h}$, and real-time growth kinetics monitored for one week. MDA-MB231 cells overexpressing CPT1A had an approximately $20-25 \%$ lower confluency than controls (paired t-test $p<0.05)$ in two independently generated pTRE-CPT1A cell lines (Fig. 7). This effect was not attributable to Dox treatment per se, as the control TetOn parental line had similar growth rates in the presence or absence of Dox (Figs 7b and c).

Overexpression of CPT1A decreases wound healing rate in MDA-MB231 cell line - We also investigated whether CPT1A overexpression altered gap closure rate in MDA-MB231 cells. Cells were induced for five days to express CPT1A and then seeded into 96-well plate at full 


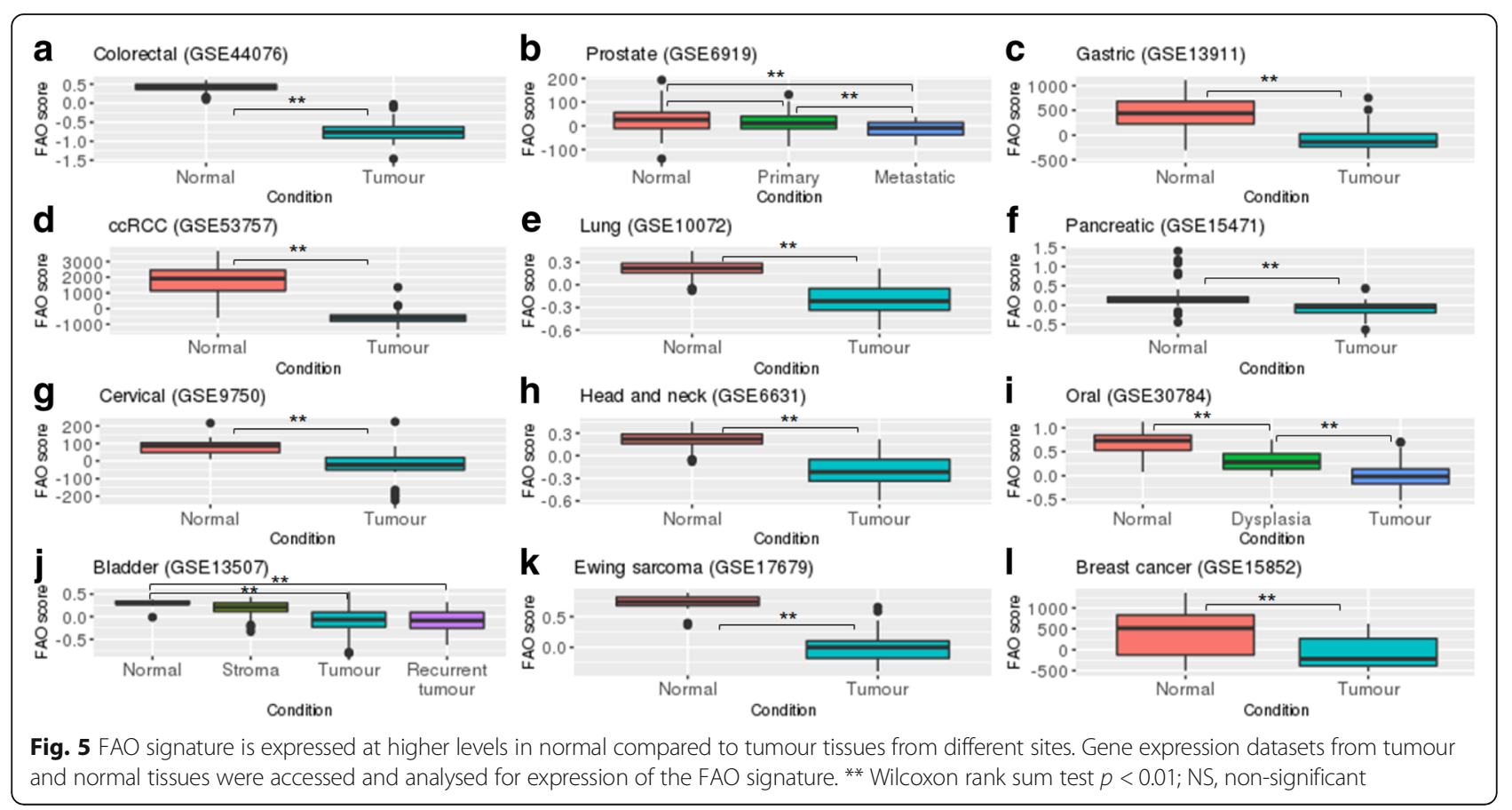

confluency. A scratch was made through the middle of each well and gap closure rates between control and CPT1A overexpressing cells were monitored. As shown in Fig. 8, the wound closure rate was up to $16 \%$ slower in cells with CPT1A overexpression, compared to control (paired t-test $p<0.05$ ). No significant difference in wound healing migration was observed between the Tet parental line in the presence or absence of Dox (Fig. 8b, c). Representative phase contrast images are shown in Additional file 6: Fig. S4.

Overexpression of CPT1A does not influence clonogenic growth in MDA-MB231 cell line - To investigate whether CPT1A overexpression in MDA-MB231 cells affects anchorage-independent clonogenic growth, we seeded and cultured cells in soft agar for two weeks, and counted colonies from each condition. As shown in Fig. 9, no significant difference in the number of colonies was observed between basal and CPT1A overexpressing MDA-MB231 cells.

\section{Discussion}

This study sought to investigate genes and pathways that are associated with prognosis in breast cancer. Our bioinformatic analysis identified a gene expression signature composed of genes associated with FAO that was correlated with survival in some cancers. Cancer metabolism is an emerging hallmark of cancer [4]. Many studies have focused on understanding how glycolysis, glutamine metabolism and fatty acid synthesis affect cancer cell biology [7-9], however, the role of FAO in cancer remains contentious. Breast cancer cells treated with etomoxir to inhibit CPT1A resulted in cell death, while in vivo, mutant KRAS lung tumours were dependent on ACSL3-dependent FAO for tumour initiation and progression [36, 37]. However, findings from recent studies focused on human tumour samples and in vivo animal models suggest that activating FAO negatively affects tumour growth and progression [38, 39]. Here, we report a prognostic association between the expression of a gene signature involved in FAO and prognosis in some cancers. High expression of this signature was associated with better survival; and we validated this finding in multiple independent breast cancer datasets.

We observed that the FAO signature was expressed more highly in ER-positive and luminal molecular subtype breast tumours. This may be a result of the negative correlation between proliferation and the FAO signature expression, as ER-negative and basal/HER2-enriched molecular subtype tumours are generally more proliferative than ER-positive/luminal subtype tumours. However, Louie et al. previously demonstrated that the ER-negative MDA-MB231 cells incorporate exogenous palmitate into structural and signalling lipids, while the ER-positive MCF7 cells direct exogenous palmitate into acyl-carnitine - precursors of beta oxidation [40]. The different fates in response to exogenous palmitate may also explain why the FAO signature expression is lower in ER-negative, compared to ER-positive tumours.

In the neoadjuvant treatment setting, we found that ER-positive tumours from patients with high FAO signature expression had a better response to short-term 

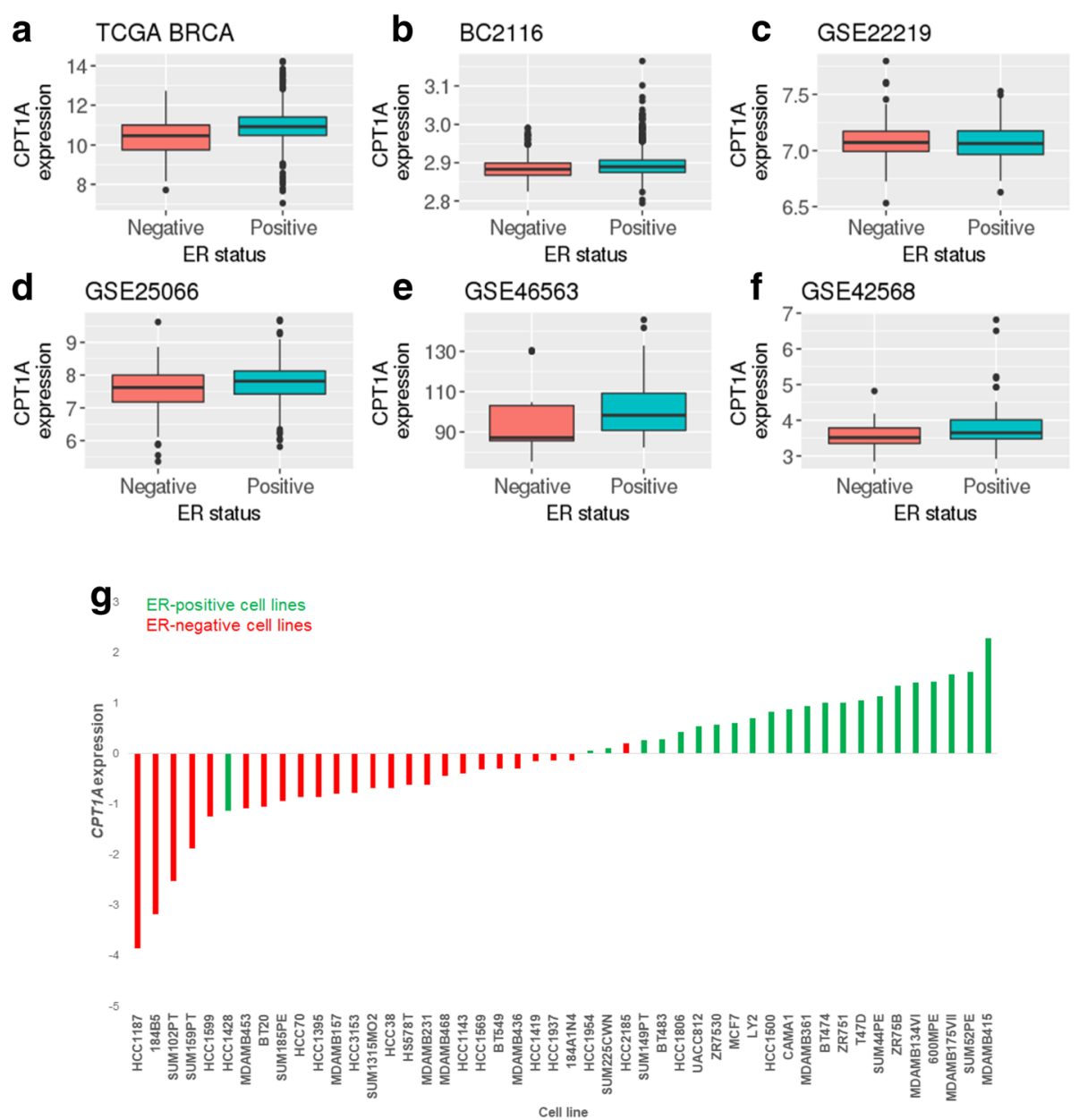

Fig. 6 Expression of CPT1A is expressed at higher levels in ER-positive, compared to ER-negative breast tumours and cell lines. a-f CPT1A is expressed higher in ER-positive than ER-negative tumours in 5 of 6 datasets analysed. $\mathbf{g}$-h In two independent datasets, ER-positive (green bars) breast cancer cell lines generally exhibit higher CPT1A expression, compared to ER-negative (red bars) cell lines. ER-positive vs ER-negative Wilcoxon rank sum test $p<0.01$ for both datasets. ${ }^{* *}$ Wilcoxon rank sum test $p<0.01$

oestrogen deprivation therapy. Additionally, patients with tumours that had low FAO signature expression that received pre-surgical chemotherapy had better odds of achieving complete response. The inverse correlation between the FAO signature expression and proliferation may explain why tumours with low expression of the FAO signature are more likely to achieve good chemotherapy response. In support of this finding, we analysed publicly available gene expression data from MCF7 cells with knockdown of ER expression, and observed higher expression of the MKS signature, and a trend towards decreased expression of the FAO signature, in cells with ER knockdown compared to control (Additional file 7: Fig. S5). Hence, these findings suggest a robust inverse relationship between cancer cell proliferation and the FAO signature expression.

We demonstrate in multiple tumour types that the FAO signature expression is downregulated in tumour, compared to normal, non-tumour tissues. In some tissue types, these findings are supported by the literature. In gastric cancer, Enjoji et al. performed a pilot analysis by qPCR and reported the expression of genes involved in FAO to be downregulated in tumours, compared to normal gastric tissues [41]. La Gory et al. reported that the expression of genes involved in FAO was lower in the 786-O ccRCC cell line, compared to normal kidney cells [42] and, recently, Du et al. reported that CPT1A expression is decreased in ccRCC versus normal kidney [43]. Here, we demonstrate that the FAO signature expression is lower in ccRCC compared to normal kidney tissues in multiple datasets. In colorectal cancer, proteomic profiling of normal colorectal tissue, benign adenoma and colorectal carcinoma found that the expression of enzymes involved in FAO were downregulated with advanced disease [44]. This finding supports our observation that the FAO signature expression is lower in colorectal carcinomas, compared to normal colorectal tissues. Notably, 

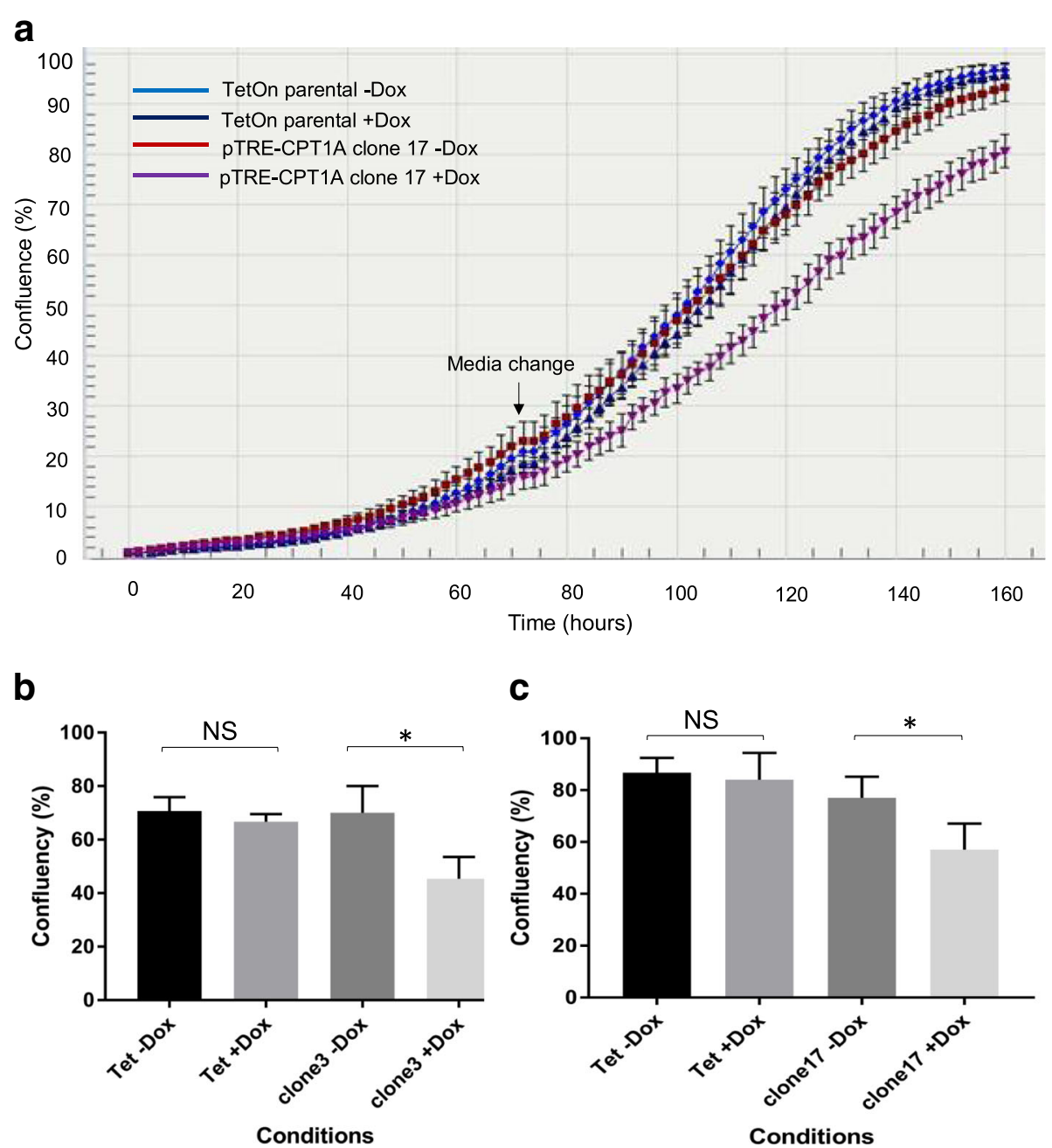

Fig. 7 CPT1A overexpression decreases confluency of MDA-MB231 cell line. a Representative growth curve of MDA-MB231 pTRE-CPT1A cell lines with endogenous (blue circle) and CPT1A overexpression (navy blue triangle). Error bars = standard deviation. $\mathbf{b}, \mathbf{c}$ In two independently generated MDA-MB231 pTRE-CPT1A b clone 3 and $\mathbf{c}$ clone 17, CPT1A overexpression decreased confluency at $120 \mathrm{~h}(\mathrm{n}=3$, error bars = standard error of mean; * paired t-test -Dox vs + Dox $p<0.05$; NS = non-significant)

our data suggests a correlation between transcript abundance and the expression level of enzymes involved in FAO. Our finding is also consistent with the observation by Gaude et al. who reported a gene set involved in FAO to be downregulated in tumour, relative to normal tissues in $30 \%$ of cancer types examined [45]. Additionally, we observed the same trend in several other tumour types including lung, pancreatic, bladder and stomach adenocarcinomas, and oesophageal and renal cell carcinomas, which were not reported in the Gaude et al. study. Taken together, our analysis has shown the downregulation of the FAO signature expression in a broader range of tumour-normal tissues relative to that reported in the literature.

Expression of CPT1A - the gene encoding the enzyme catalysing the rate-limiting step in FAO - was lower in ER-negative, compared to ER-positive tumours in most of the datasets analysed. Furthermore, in silico analysis of gene expression data from a panel of breast cancer cell lines found that CPT1A expression was higher in ER-positive, compared to ER-negative cell lines. This is supported by the findings of Balaban et al., who reported the rate of oxidation of radiolabelled palmitate to be over 5 -fold higher in MCF7 cells compared to the MDA-MB231 cell line [46]. It is important to note that in addition to major differences in ER expression between these two cell lines, they also have unique genetic aberration profiles [47]. Therefore, one can posit that changes in CPT1A expression may not just be driven by specific mutations or other genomic events, but rather, are part of the broader alterations that occur during tumour initiation and progression.

Overexpression of CPT1A in MDA-MB231 cells decreased their confluency by approximately $20-25 \%$, and wound healing rate by $16 \%$. These data suggest that modulating the rate-limiting enzyme of FAO can significantly 
a

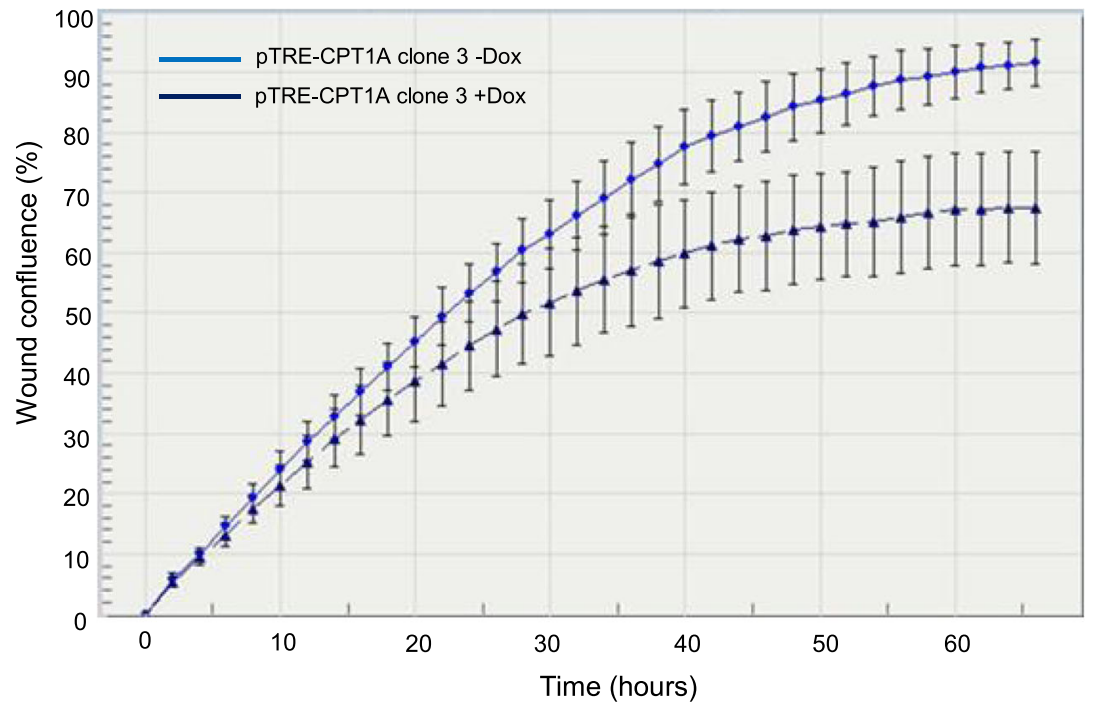

b

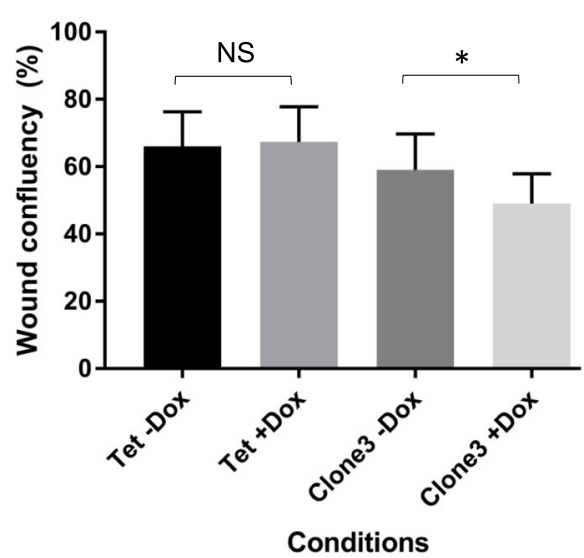

C

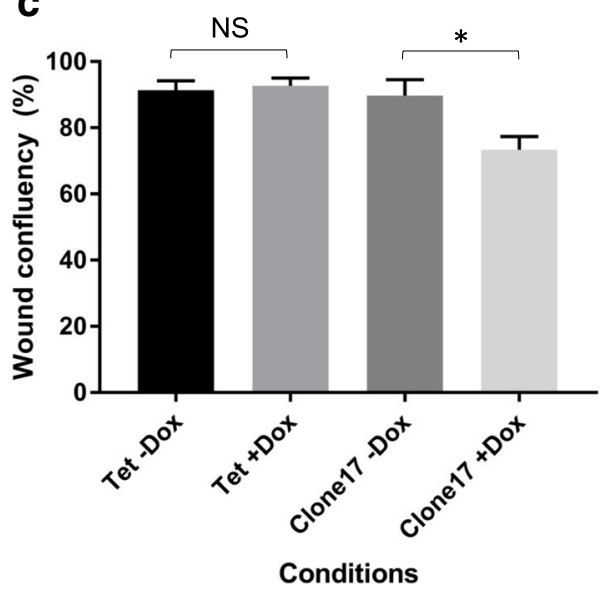

Fig. 8 CPT1A overexpression decreases wound healing migration of MDA-MB231 cell line. a Representative wound closure curve of MDA-MB231 PTRE-CPT1A cell lines with endogenous (blue circle) and CPT1A overexpression (navy blue triangle). Error bars = standard deviation. $\mathbf{b}, \mathbf{c}$ In two independently generated MDA-MB231 PTRE-CPT1A $\mathbf{b}$ clone 3 and $\mathbf{c}$ clone 17, CPT1A overexpression decreased wound closure rate $(n=3$, error bars = standard error of mean; ${ }^{*}$ paired t-test -Dox vs + Dox $p<0.05 ;$ NS = non-significant)

alter the proliferation and migration rates of cancer cells. This decreased proliferation in MDA-MB231 cells in response to CPT1A overexpression is supported by two studies that modulate FAO by different means. Treatment of lung cancer cell lines with pioglitazone - an agonist of the nuclear receptor peroxisome proliferator activated receptor gamma - decreased the proliferation rates via increased FAO; which activated the tumour suppressor protein retinoblastoma to effect cell cycle arrest. In prostate tumours, Torrano et al. demonstrated that higher expression of PPARGC1A - a key transcriptional co-regulator that activates expression of genes involved in oxidative metabolism - was associated with favourable prognosis [39]. Importantly, overexpression of PGC1A (the protein encoded by PPARGC1A) increased FAO flux in PC3 prostate cancer cells, and decreased their proliferation and soft agar clonogenic growth. In another study, FAO flux analysis of RWPE-1 prostate epithelial cells and its increasingly invasive derivative lines found decreased FAO with increasing invasiveness [48]. Overexpression of CPT1A in MDA-MB231, however, did not affect clonogenic growth of the cells, compared to the control. One plausible explanation for this observation is that growth of cells under anchorage-independent conditions alter their metabolic requirements, which may differ from two-dimensional cell growth $[49,50]$. Indeed, growth of the normal mammary epithelial MCF10A cells was impaired in part due to decreased glycolysis, and could be rescued by activation of FAO [49].

We acknowledge that our study is limited by the number of cell lines analysed for experimental characterisation of the bioinformatics findings. As such, future efforts could focus on modulating this pathway in cell lines from 


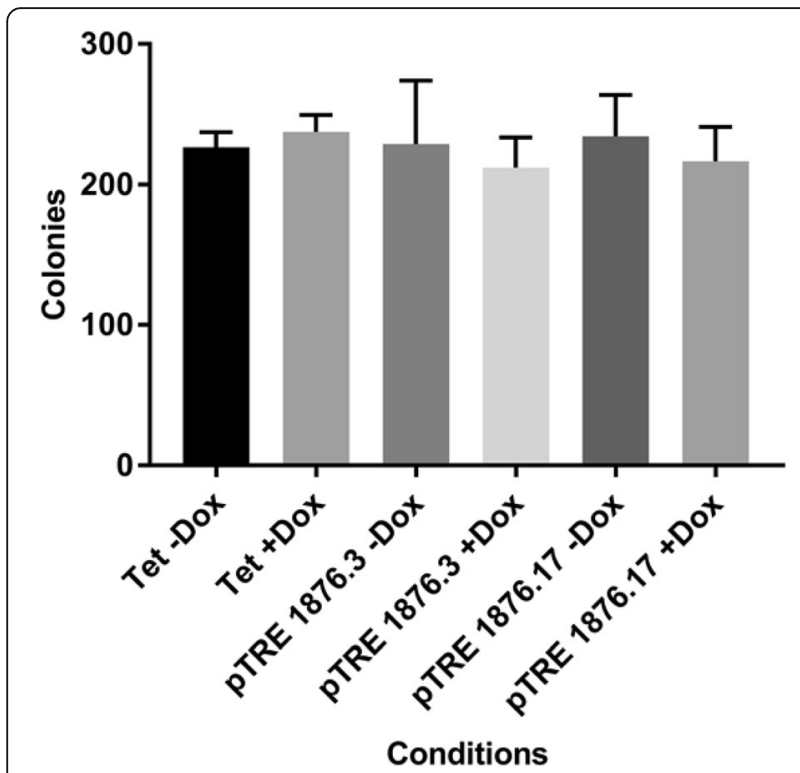

Fig. 9 CPT1A overexpression does not alter anchorage-independent growth of MDA-MB231 cells. MDA-MB231 pTRE-CPT1A and TetOn parental lines were cultured in agar for two weeks and colonies counted under phase contrast. Number of colonies between -Dox and + Dox for TetOn parental and PTRE-CPT1A clones 3 and 17 were not statistically significant. $n=3$, error bars $=$ standard error of mean

breast, as well as other cancer types, which could shed light on the common mechanisms as to how alterations in FAO affects cancer cell biology.

\section{Conclusions}

In this study, we report an association between expression of genes involved in FAO and prognosis across multiple tumour types. Overexpression of CPT1A - the rate-limiting enzyme in FAO - decreased the proliferation rate of MDA-MB231 breast cancer cells. Hence, activation of this pathway in the adjuvant setting may improve treatment outcome, and future studies could explore the effect of modulating FAO in other cancers where the FAO signature has been shown to predict survival.

\section{Additional files}

Additional file 1: Table S1. Published gene expression datasets analysed in this study. (XLSX $10 \mathrm{~kb})$

Additional file 2: Table S2. FAO signature expression and outcome in colorectal cancer datasets. (XLSX $10 \mathrm{~kb}$ )

Additional file 3: Figure S1. FAO signature expression is not associated with ovarian cancer overall survival from the KMplotter analysis. (PDF $180 \mathrm{~kb}$ )

Additional file 4: Figure S2. Expression of CPT1A lower in ER-negative, relative to ER-positive breast cancer cell lines. Black arrow indicates an adriamycin-resistant MCF7 cell line, which had lower CPT1A expression, compared to wild type MCF7 cells. (PDF $90 \mathrm{~kb}$ )

Additional file 5: Figure S3. Representative dose response and time course analysis of doxycycline induction of CPT1A expression in MDAMB231 cells. (a) Dose response optimisation of doxycycline induction of
CPT1A. Cells were seeded and induced with increasing concentrations of Dox from 0 - to $5 \mu \mathrm{g} / \mathrm{mL}$ for $48 \mathrm{~h}$. Twenty $\mu \mathrm{g}$ of proteins were resolved by SDS-PAGE, and immunoblotted for CPT1A expression. (b) Time course optimisation of Dox induction. PTRE-CPT1A clone 3 cells were seeded, induced with $2 \mu \mathrm{g} / \mathrm{mL}$ Dox for up to $96 \mathrm{~h}$, and immunoblotted for CPT1A expression. (PDF $47 \mathrm{~kb}$ )

Additional file 6: Figure S4. Representative wound healing migration phase contrast images. The scratch wound is completely closed at $30 \mathrm{~h}$ in MDA-MB231 TetOn parental cells with Dox (a) or without (b) treatment. (c) The wound area in PTRE-CPT1A clone 17 -Dox clones were completely closed at $30 \mathrm{~h}$, but not (d) cells induced with Dox. (PDF $117 \mathrm{~kb}$ )

Additional file 7: Figure S5. ER knockdown in MCF7 cells decrease FAO signature expression. MCF7 cells stably expressing shRNA against ESR1 (gene encoding ER) had decreased expression of the (a) FAO signature, but increased expression of the (b) MKS proliferation signature. $n=3$ for both basal and ESR1 knockdown. ${ }^{* *}$ t-test $p<0.01$. (PDF $66 \mathrm{~kb}$ )

\section{Abbreviations}

ACADM/SB/NL: Acyl-CoA dehydrogenase medium/short-branched/very long chain; ACSL3: Acyl-CoA synthetase long chain 3; ccRCC: Clear cell renal cell carcinoma; CoA: Coenzyme A; CPT1A: Carnitine palmitoyl transferase 1A; CPT2: Carnitine palmitoyl transferase 2; Dox: Doxycycline; ER: Oestrogen Receptor; FAIMoS: Functional Aromatase Inhibitor Molecular Study; FAO: Fatty acid oxidation; KEGG: Kyoto Encyclopedia of Genes and Genomes; METABRIC: Molecular Taxonomy of Breast Cancer International Consortium; MKS: Mitosis kinome score; pCR: Pathological complete response; PPARGC1A/PGC1A: Peroxisome proliferator-activated gamma coactivator 1A; qPCR: Quantitative polymerase chain reaction; RECIST: Response Evaluation Criteria in Solid Tumours

\section{Funding}

AA is a recipient of the University of Otago Doctoral Scholarship. AD was supported by a New Zealand Health Research Council Sir Charles Hercus Fellowship. No specific funding was received for this study and the individual funding bodies listed above played no part in the design of the study and collection, analysis, and interpretation of data or in writing the manuscript.

\section{Availability of data and materials}

All data generated during this study are included in this published article [and its supplementary information files]. Gene expression data and clinical information from the METABRIC study was obtained through Sage Bionetworks (sagebase.org/). Other gene expression data sets were obtained from Gene Expression Ominibus (https://www.ncbi.nlm.nih.gov/geo/) and the Genomic Data Commons (https://portal.gdc.cancer.gov/).

\section{Authors' contributions}

AA designed, performed and analysed all the bioinformatics and experimental analyses, and wrote the manuscript. MAB provided input on the design of survival analysis of the training data, supervised bioinformatics and statistical analyses, and reviewed the manuscript. AKD supervised this study and wrote the manuscript. All authors reviewed the results and approved the final manuscript.

\section{Ethics approval and consent to participate}

Informed consent to participate was obtained from participants of the original studies as described in the papers referenced in the methods section. The original METABRIC study was approved by the ethics Institutional Review Boards in the UK and Canada (Addenbrooke's Hospital, Cambridge, United Kingdom; Guy's Hospital, London; Nottingham; Vancouver; Manitoba). Ethical approval for access to the METABRIC data was sought and granted from the University of Otago Human Ethics Committee (Approval H16/092).

\section{Consent for publication}

Not applicable.

\section{Competing interests}

The authors declare that they have no competing interests. 


\section{Publisher's Note}

Springer Nature remains neutral with regard to jurisdictional claims in published maps and institutional affiliations.

Received: 8 January 2018 Accepted: 21 June 2018

Published online: 09 August 2018

\section{References}

1. Kittaneh M, Montero AJ, Gluck S. Molecular profiling for breast cancer: a comprehensive review. Biomarkers in Cancer. 2013;5:61-70.

2. Weigelt B, Pusztai L, Ashworth A, Reis-Filho JS. Challenges translating breast cancer gene signatures into the clinic. Nat Rev Clin Oncol. 2011;9(1):58-64.

3. Wirapati $P$, Sotiriou $C$, Kunkel S, Farmer P, Pradervand S, Haibe-Kains B, Desmedt C, Ignatiadis M, Sengstag T, Schutz F, et al. Meta-analysis of gene expression profiles in breast cancer: toward a unified understanding of breast cancer subtyping and prognosis signatures. Breast Cancer Res : BCR. 2008;10(4):R65.

4. Hanahan D, Weinberg RA. Hallmarks of cancer: the next generation. Cell. 2011;144(5):646-74.

5. Ward PS, Thompson CB. Metabolic reprogramming: a cancer hallmark even Warburg did not anticipate. Cancer Cell. 2012;21(3):297-308.

6. Koppenol WH, Bounds PL, Dang CV. Otto Warburg's contributions to current concepts of cancer metabolism. Nat Rev Cancer. 2011;11(5):325-37.

7. Lunt SY, Vander Heiden MG. Aerobic glycolysis: meeting the metabolic requirements of cell proliferation. Annu Rev Cell Dev Biol. 2011;27:441-64.

8. Altman BJ, Stine ZE, Dang CV. From Krebs to clinic: glutamine metabolism to cancer therapy. Nat Rev Cancer. 2016;16(11):749.

9. Rohrig F, Schulze A. The multifaceted roles of fatty acid synthesis in cancer. Nat Rev Cancer. 2016;16(11):732-49.

10. Curtis C, Shah SP, Chin SF, Turashvili G, Rueda OM, Dunning MJ, Speed D, Lynch AG, Samarajiwa S, Yuan Y, et al. The genomic and transcriptomic architecture of 2,000 breast tumours reveals novel subgroups. Nature. 2012; 486(7403):346-52.

11. R Development Core team. R: a language and environment for statistical computing. In: R Foundation for Statistical Computing; 2017.

12. Miller JA, Cai C, Langfelder P, Geschwind DH, Kurian SM, Salomon DR, Horvath S. Strategies for aggregating gene expression data: the collapseRows R function. BMC Bioinf. 2011;12:322.

13. Therneau TM, Grambsch PM. Modeling survival data : extending the cox model. New York; London: Springer; 2011.

14. Benjamini $Y$, Hochberg $Y$. Controlling the false discovery rate: a practical and powerful approach to multiple testing. J R Stat Soc Ser B Methodol. 1995;57(1):289-300.

15. Subramanian A, Tamayo P, Mootha VK, Mukherjee S, Ebert BL, Gillette MA, Paulovich A, Pomeroy SL, Golub TR, Lander ES, et al. Gene set enrichment analysis: a knowledge-based approach for interpreting genome-wide expression profiles. Proc Natl Acad Sci U S A. 2005;102(43):15545-50.

16. Kanehisa M, Goto S. KEGG: Kyoto encyclopedia of genes and genomes. Nucleic Acids Res. 2000;28(1):27-30.

17. Lanczky A, Nagy A, Bottai G, Munkacsy G, Szabo A, Santarpia L, Gyorffy B. miRpower: a web-tool to validate survival-associated miRNAs utilizing expression data from 2178 breast cancer patients. Breast Cancer Res Treat. 2016;160(3):439-46.

18. Bengtsson $H$, Wirapati $P$, Speed TP. A single-array preprocessing method for estimating full-resolution raw copy numbers from all Affymetrix genotyping arrays including GenomeWideSNP 5 \& 6. Bioinfor (Oxford, England). 2009; 25(17):2149-56.

19. Heiser LM, Sadanandam A, Kuo WL, Benz SC, Goldstein TC, Ng S, Gibb WJ, Wang NJ, Ziyad S, Tong F, et al. Subtype and pathway specific responses to anticancer compounds in breast cancer. Proc Natl Acad Sci U S A. 2012; 109(8):2724-9.

20. Bolstad BM, Irizarry RA, Astrand M, Speed TP. A comparison of normalization methods for high density oligonucleotide array data based on variance and bias. Bioinfor (Oxford, England). 2003;19(2):185-93.

21. van Diest PJ, van der Wall E, Baak JP. Prognostic value of proliferation in invasive breast cancer: a review. J Clin Pathol. 2004;57(7):675-81.

22. Bianchini G, Pusztai L, Karn T, Iwamoto T, Rody A, Kelly C, Muller V, Schmidt S, Qi Y, Holtrich U, et al. Proliferation and estrogen signaling can distinguish patients at risk for early versus late relapse among estrogen receptor positive breast cancers. Breast Cancer Res : BCR. 2013;15(5):R86.
23. Houten SM, Wanders RJ. A general introduction to the biochemistry of mitochondrial fatty acid beta-oxidation. J Inherit Metab Dis. 2010;33(5):469-77.

24. Smith IE, Walsh G, Skene A, Llombart A, Mayordomo JI, Detre S, Salter J, Clark E, Magill P, Dowsett M. A phase II placebo-controlled trial of neoadjuvant anastrozole alone or with gefitinib in early breast cancer. J Clin Oncol. 2007:25(25):3816-22.

25. Iwamoto T, Bianchini G, Booser D, Qi Y, Coutant C, Shiang CY, Santarpia L, Matsuoka J, Hortobagyi GN, Symmans WF, et al. Gene pathways associated with prognosis and chemotherapy sensitivity in molecular subtypes of breast cancer. J Natl Cancer Inst. 2011;103(3):264-72.

26. Hatzis C, Pusztai L, Valero V, Booser DJ, Esserman L, Lluch A, Vidaurre T, Holmes F, Souchon E, Wang $H$, et al. A genomic predictor of response and survival following taxane-anthracycline chemotherapy for invasive breast cancer. JAMA. 2011;305(18):1873-81.

27. Itoh M, Iwamoto T, Matsuoka J, Nogami T, Motoki T, Shien T, Taira N, Niikura N, Hayashi N, Ohtani S, et al. Estrogen receptor (ER) mRNA expression and molecular subtype distribution in ER-negative/progesterone receptorpositive breast cancers. Breast Cancer Res Treat. 2014;143(2):403-9.

28. Miyake T, Nakayama T, Naoi Y, Yamamoto N, Otani Y, Kim SJ, Shimazu K, Shimomura A, Maruyama N, Tamaki Y, et al. GSTP1 expression predicts poor pathological complete response to neoadjuvant chemotherapy in ERnegative breast cancer. Cancer Sci. 2012;103(5):913-20.

29. Shen K, Qi Y, Song N, Tian C, Rice SD, Gabrin MJ, Brower SL, Symmans WF, O'Shaughnessy JA, Holmes FA, et al. Cell line derived multi-gene predictor of pathologic response to neoadjuvant chemotherapy in breast cancer: a validation study on US oncology 02-103 clinical trial. BMC Med Genet. 2012;5:51.

30. Hess KR, Anderson K, Symmans WF, Valero V, Ibrahim N, Mejia JA, Booser D, Theriault RL, Buzdar AU, Dempsey PJ, et al. Pharmacogenomic predictor of sensitivity to preoperative chemotherapy with paclitaxel and fluorouracil, doxorubicin, and cyclophosphamide in breast cancer. J Clin Oncol. 2006; 24(26):4236-44.

31. Jambor de Sousa UL, Koss MD, Fillies M, Gahl A, Scheeder MR, Cardoso MC, Leonhardt H, Geary N, Langhans W, Leonhardt M. CPT1alpha overexpression increases long-chain fatty acid oxidation and reduces cell viability with incremental palmitic acid concentration in 293T cells. Biochem Biophys Res Commun. 2005;338(2):757-61.

32. Pike LS, Smift AL, Croteau NJ, Ferrick DA, Wu M. Inhibition of fatty acid oxidation by etomoxir impairs NADPH production and increases reactive oxygen species resulting in ATP depletion and cell death in human glioblastoma cells. Biochim Biophys Acta. 2011;1807(6):726-34.

33. Deberardinis RJ, Lum JJ, Thompson CB. Phosphatidylinositol 3-kinasedependent modulation of carnitine palmitoyltransferase $1 \mathrm{~A}$ expression regulates lipid metabolism during hematopoietic cell growth. J Biol Chem. 2006:281(49):37372-80.

34. Gossen M, Freundlieb S, Bender G, Muller G, Hillen W, Bujard H. Transcriptional activation by tetracyclines in mammalian cells. Science. 1995; 268(5218):1766-9.

35. Gossen M, Bujard $H$. Tight control of gene expression in mammalian cells by tetracycline-responsive promoters. Proc Natl Acad Sci U S A. 1992:89(12):5547-51.

36. Hardy S, El-Assaad W, Przybytkowski E, Joly E, Prentki M, Langelier Y. Saturated fatty acid-induced apoptosis in MDA-MB-231 breast cancer cells. A role for cardiolipin J Biol Chem. 2003;278(34):31861-70.

37. Padanad MS, Konstantinidou G, Venkateswaran N, Melegari M, Rindhe S, Mitsche M, Yang C, Batten K, Huffman KE, Liu J, et al. Fatty acid oxidation mediated by acyl-CoA Synthetase long chain 3 is required for mutant KRAS lung tumorigenesis. Cell Rep. 2016;16(6):1614-28.

38. Srivastava N, Kollipara RK, Singh DK, Sudderth J, Hu Z, Nguyen H, Wang S, Humphries CG, Carstens R, Huffman KE, et al. Inhibition of cancer cell proliferation by PPARgamma is mediated by a metabolic switch that increases reactive oxygen species levels. Cell Metab. 2014;20(4):650-61.

39. Torrano V, Valcarcel-Jimenez L, Cortazar AR, Liu X, Urosevic J, Castillo-Martin M, Fernandez-Ruiz S, Morciano G, Caro-Maldonado A, Guiu M, et al. The metabolic co-regulator PGC1alpha suppresses prostate cancer metastasis. Nat Cell Biol. 2016;18(6):645-56.

40. Louie SM, Roberts LS, Mulvihill MM, Luo K, Nomura DK. Cancer cells incorporate and remodel exogenous palmitate into structural and oncogenic signaling lipids. Biochim Biophys Acta. 2013;1831(10):1566-72.

41. Enjoji M, Kohjima M, Ohtsu K, Matsunaga K, Murata $Y$, Nakamuta M, Imamura K, Tanabe H, Iwashita A, Nagahama T, et al. Intracellular 
mechanisms underlying lipid accumulation (white opaque substance) in gastric epithelial neoplasms: a pilot study of expression profiles of lipidmetabolism-associated genes. J Gastroenterol Hepatol. 2016;31(4):776-81.

42. LaGory EL, Wu C, Taniguchi CM, Ding CK, Chi JT, von Eyben R, Scott DA, Richardson AD, Giaccia AJ. Suppression of PGC-1alpha is critical for reprogramming oxidative metabolism in renal cell carcinoma. Cell Rep. 2015;12(1):116-27.

43. Du W, Zhang L, Brett-Morris A, Aguila B, Kerner J, Hoppel CL, Puchowicz M, Serra D, Herrero L, Rini BI, et al. HIF drives lipid deposition and cancer in CCRCC via repression of fatty acid metabolism. Nat Commun. 2017;8(1):1769.

44. Wisniewski JR, Dus-Szachniewicz K, Ostasiewicz P, Ziolkowski P, Rakus D, Mann M. Absolute proteome analysis of colorectal mucosa, adenoma, and Cancer reveals drastic changes in fatty acid metabolism and plasma membrane transporters. J Proteome Res. 2015;14(9):4005-18.

45. Gaude E, Frezza C. Tissue-specific and convergent metabolic transformation of cancer correlates with metastatic potential and patient survival. Nat Commun. 2016:7:13041.

46. Balaban S, Shearer RF, Lee LS, van Geldermalsen M, Schreuder M, Shtein HC, Cairns R, Thomas KC, Fazakerley DJ, Grewal T, et al. Adipocyte lipolysis links obesity to breast cancer growth: adipocyte-derived fatty acids drive breast cancer cell proliferation and migration. Cancer \& Metab. 2017;5(1)

47. Hollestelle A, Nagel JH, Smid M, Lam S, Elstrodt F, Wasielewski M, Ng SS, French PJ, Peeters JK, Rozendaal MJ, et al. Distinct gene mutation profiles among luminal-type and basal-type breast cancer cell lines. Breast Cancer Res Treat. 2010;121(1):53-64.

48. Vayalil PK, Landar A. Mitochondrial oncobioenergetic index: a potential biomarker to predict progression from indolent to aggressive prostate cancer. Oncotarget. 2015;6(40):43065-80.

49. Schafer ZT, Grassian AR, Song L, Jiang Z, Gerhart-Hines Z, Irie HY, Gao S, Puigserver $P$, Brugge JS. Antioxidant and oncogene rescue of metabolic defects caused by loss of matrix attachment. Nature. 2009;461(7260):109-13.

50. Jiang L, Shestov AA, Swain P, Yang C, Parker SJ, Wang QA, Terada LS, Adams ND, McCabe MT, Pietrak B, et al. Reductive carboxylation supports redox homeostasis during anchorage-independent growth. Nature. 2016; 532(7598):255-8.

\section{Ready to submit your research? Choose BMC and benefit from:}

- fast, convenient online submission

- thorough peer review by experienced researchers in your field

- rapid publication on acceptance

- support for research data, including large and complex data types

- gold Open Access which fosters wider collaboration and increased citations

- maximum visibility for your research: over $100 \mathrm{M}$ website views per year

At BMC, research is always in progress.

Learn more biomedcentral.com/submissions 